\title{
DSmT qualitative reasoning based on 2-Tuple linguistic representation model
}

\author{
Xinde Li and Xianzhong Dai \\ Key Laboratory of Measurement and Control of CSE \\ Ministry of Education, \\ Nanjing, China, 210096 \\ xindeli@seu.edu.cn
}

\author{
Jean Dezert \\ French Aerospace Research Lab. \\ 29 Avenue de la Division Leclerc \\ 92320 Châtillon, France \\ jean.dezert@onera.fr
}

\author{
Florentin Smarandache \\ Chair of Math. \& Sciences Dept. \\ University of New Mexico, \\ 200 College Road, Gallup, NM 87301, U.S.A. \\ smarand@unm.edu
}

\begin{abstract}
Most of modern systems for information retrieval, fusion and management have to deal more and more with information expressed quatitatively (by linguistic labels) since human reports are better and easier expressed in natural language than with numbers. In this paper, we propose to use Herrera-Martínez' 2-Tuple linguistic representation model (i.e. equidistant linguistic labels with a numeric value assessment) for reasoning with uncertain and qualitative information in Dezert-Smarandache Theory (DSmT) framework to preserve the precision and the efficiency of the fusion of linguistic information expressing the expert's qualitative beliefs. We present operators to deal with the 2-Tuples and show from a simple example how qualitative DSmT-based fusion rules can be used for qualitative reasoning and fusioning under uncertainty.
\end{abstract}

Keywords: DSmT, Dezert-Smarandache Theory, Information fusion, Qualitative reasoning, linguistic labels.

\section{Introduction}

Qualitative methods for reasoning under uncertainty have gained more and more attention because traditional methods based only on quantitative representation and analysis are not able to adequately satisfy the need of the development of science and technology integrating at higher fusion levels human beliefs and reports in complex systems. Therefore qualitative knowledge representation and analysis becomes important and necessary in next genera- tions of decision-making support systems. Most of existing approaches use the 1-Tuple linguistic representation model consisting in a given finite ordered set of pure linguistic labels, say $L=\left\{L_{0}, \tilde{L}, L_{n+1}\right\}$ where $\tilde{L}=\left\{L_{1}, \cdots, L_{n}\right\}$. Smarandache and Dezert give a detailed introduction of major works for 1-Tuple qualitative reasoning under uncertainty in [5] and also propose new well-justified operators on 1-Tuple labels in [1]. They have also shown how quantitative combination rules can be easily extended for dealing with qualitative beliefs represented in terms of pure linguistic labels (i.e. 1-Tuple). In order to keep an acceptable computational complexity it is obviously better to work with a reduced/coarse granularity set of pure linguistic labels (1Tuple) but by doing so, some available richer information content, like less good, good enough, very good, is lost in the classical/1-Tuple qualitative information processing. To overcome this limitation, Herrera and Martínez in [2] proposed a 2-Tuple fuzzy linguistic representation model for computing with words $(\mathrm{CW})$, which offers a computationally feasible method for aggregating linguistic information that are represented by linguistic variables with equidistant labels ${ }^{1}$ through counting indexes of the corresponding linguistic labels. It has been shown in [2] the advantages of the 2-Tuple Linguistic representation of symbolic method over methods based on the extension principle in $\mathrm{CW}$ in term of complexity and feasibility. In 2007, Li et al. [3] have proposed in the DSmT framework, the extension of 1-Tuple linguistic representation model to Qualitative En-

\footnotetext{
${ }^{1}$ For non-equidistant labels we can still use these 2-Tuples labels in the same way, but of course the result is less accurate, yet giving a satisfactory result.
} 
riched labels, denoted $L_{i}\left(\sigma_{i}^{e}\right)$, for taking into account a possible confidence factor $\sigma_{i}^{e}$ on any label $L_{i}$. In this paper, we propose to use Herrera's 2-Tuple representation denoted $\left(L_{i}, \sigma_{i}^{h}\right)$ besides our previous 1-Tuple (enriched) representation where $\sigma_{i}^{h}$ expresses a kind of refinement of the linguistic value $L_{i}$. Clearly $\sigma_{i}^{e}$ and $\sigma_{i}^{h}$ correspond to two distinct notions. In section 2, we remind briefly the basics of DSmT. In sections 3 and 4, we recall the 1-Tuple models (classical and enriched). In sections 5 and 6, we present the 2-Tuple model together with the basic operators for 2-Tuple labels. Qualitative fusion rules based on 2-Tuple linguistic labels are proposed in section 7 . In section 8 , we show how these operators are used for combining 2-Tuple qualitative beliefs. Concluding remarks are then given in section 9 .

\section{DSmT for the fusion of beliefs}

\section{$2.1 \quad$ Basic belief mass}

The differences between Dempster-Shafer Theory (DST) [4], and Dezert-Smarandache Theory (DSmT) [5] are:

1. The model on which one works with. Typically if one considers a finite frame of possible exhaustive solutions $\Theta=\left\{\theta_{1}, \ldots, \theta_{n}\right\}$, Shafer assumes the exclusivity of $\theta_{i}$ and defines belief masses on classical power set $2^{\Theta} \triangleq(\Theta, \cup)$ while we don't need such assumption in DSmT and the belief masses can be defined directly on Dedekind's lattice/hyper-power set $D^{\Theta} \triangleq(\Theta, \cup, \cap)$ and even on the super-power set $S^{\Theta} \triangleq(\Theta, \cup, \cap, c()$.$) if one really needs/wants to work$ on the refined frame $\Theta_{\text {ref }}$ of $\Theta$. In the sequel, we use the generic notation $G^{\Theta}$ for denoting either $2^{\Theta}, D^{\Theta}$ or $S^{\Theta}$. A quantitative basic belief assignment (bba) is a mapping $m():. G^{\Theta} \rightarrow[0,1]$ associated to a given body of evidence $\mathcal{B}$ which satisfies $m(\emptyset)=0$ and $\sum_{A \in G^{\Theta}} m(A)=1$.

2. The choice of the combination and conditioning rules, i.e. Dempster's rule in DST versus PCR5 rule in DSmT (see next section and [5] for details).

3. Aside working only with numerical/quantitative beliefs as within DST, DSmT allows also to combine directly qualitative belief masses.

\subsection{Fusion of quantitative belief masses}

In DSmT, we propose to use the Proportional Conflict Redistribution rule no. 5 (PCR5) [5, 6] which transfers conflicting masses (total or partial) proportionally to nonempty sets involved in the model according to all integrity constraints. PCR5 rule works for any degree of conflict in $[0,1]$, for any models (Shafer's model, free DSm model or any hybrid DSm model) and both in DST and DSmT frameworks for static or dynamical fusion problems. The PCR5 rule for two sources is defined by: $m_{P C R 5}(\emptyset)=0$ and $\forall X \in G^{\Theta} \backslash\{\emptyset\}$

$$
\begin{aligned}
& \sum_{\substack{Y \in G^{\Theta} \backslash\{X\} \\
X \cap Y=\emptyset}}\left[\frac{m_{1}(X)^{2} m_{2}(Y)}{m_{1}(X)+m_{2}(Y)}+\frac{m_{2}(X)^{2} m_{1}(Y)}{m_{2}(X)+m_{1}(Y)}\right]
\end{aligned}
$$

where each element $X$, and $Y$, is in the disjunctive normal form. $m_{12}(X)=\sum_{X_{1}, X_{2} \in G^{\Theta}} m_{1}\left(X_{1}\right) m_{2}\left(X_{2}\right)$ corresponds to the conjunctive consensus on $X$ between the two sources. All denominators are different from zero. If a denominator is zero, that fraction is discarded. No matter how big or small is the conflicting mass, PCR5 mathematically does a better redistribution of the conflicting mass than Dempster's rule and other rules since PCR5 goes backwards on the tracks of the conjunctive rule and redistributes the partial conflicting masses only to the sets involved in the conflict and proportionally to their masses put in the conflict, considering the conjunctive normal form of the partial conflict. PCR5 is quasi-associative and preserves the neutral impact of the vacuous belief assignment. General PCR5 fusion formula and improvement for the combination of $k \geq 2$ sources of evidence with many detailed examples can be found in [5].

\section{The 1-Tuple linguistic model}

To deal with a 1-Tuple qualitative belief over $G^{\Theta}$, one defined in [5] a qualitative basic belief assignment $q_{1} m($. as a mapping function from $G^{\Theta}$ into a set of linguistic labels $L=\left\{L_{0}, \tilde{L}, L_{n+1}\right\}$ where $\tilde{L}=\left\{L_{1}, \cdots, L_{n}\right\}$ is a finite set of linguistic labels and where $n \geq 2$ is an integer. For example, $L_{1}$ can take the linguistic value "poor", $L_{2}$ the linguistic value "good", etc. $\tilde{L}$ is endowed with a total order relationship $\prec$, so that $L_{1} \prec L_{2} \prec \cdots \prec L_{n}$. To work on a true closed linguistic set $L$ under linguistic addition and multiplication operators, Smarandache and Dezert extended naturally $\tilde{L}$ with two extreme values $L_{0}=L_{\min }$ and $L_{n+1}=L_{\max }$, where $L_{0}$ corresponds to the minimal qualitative value and $L_{n+1}$ corresponds to the maximal qualitative value, in such a way that $L_{0} \prec L_{1} \prec L_{2} \prec \cdots \prec L_{n} \prec L_{n+1}$, where $\prec$ means inferior to, or less (in quality) than, or smaller than, etc. In the sequel $L_{i} \in L$ are assumed linguistically equidistant labels such that we can make an isomorphism between $L=\left\{L_{0}, L_{1}, L_{2}, \ldots, L_{n}, L_{n+1}\right\}$ and $\{0,1 /(n+1), 2 /(n+1), \ldots, n /(n+1), 1\}$, defined as $L_{i}=i /(n+1)$ for all $i=0,1,2, \ldots, n, n+1$. 
From the extension of the isomorphism between the set of linguistic equidistant labels and a set of numbers in the interval $[0,1]$, one can built exact operators on linguistic labels which makes possible the extension all the quantitative fusion rules into their qualitative counterparts [3]. We briefly remind the basic qualitative operators ${ }^{2}$ (or $q$ operators for short) on 1-Tuple labels:

- $q$-addition:

$$
L_{i}+L_{j}= \begin{cases}L_{i+j} & \text { if } i+j<n+1 \\ L_{n+1}=L_{\max } & \text { if } i+j \geq n+1\end{cases}
$$

- $q$-subtraction:

$$
L_{i}-L_{j}= \begin{cases}L_{i-j} & \text { if } \quad i \geq j, \\ -L_{j-i} & \text { if } \quad i<j .\end{cases}
$$

where $-L=\left\{-L_{1},-L_{2}, \ldots,-L_{n},-L_{n+1}\right\}$.

- $q$-multiplication ${ }^{3}$.

$$
L_{i} \cdot L_{j}=L_{[(i \cdot j) /(n+1)]} .
$$

where $[x]$ means the closest integer ${ }^{4}$ to $x$ (with $[n+$ $0.5]=n+1, \forall n \in \mathbb{N}$ ). This operator is justified by the approximation of the product of equidistant labels given by $L_{i} \cdot L_{j}=\frac{i}{n+1} \cdot \frac{j}{n+1}=\frac{(i \cdot j) /(n+1)}{n+1}$.

- Scalar multiplication of a linguistic label: Let $a$ be a real number. The multiplication of a linguistic label by a scalar is defined by:

$$
a \cdot L_{i}=\frac{a \cdot i}{n+1} \approx \begin{cases}L_{[a \cdot i]} & \text { if }[a \cdot i] \geq 0, \\ L_{-[a \cdot i]} & \text { otherwise. }\end{cases}
$$

- Division of linguistic labels:

a) $q$-division as an internal operator: Let $j \neq 0$, then if $[(i / j) \cdot(n+1)]<n+1$ one defines

$$
L_{i} / L_{j}=L_{[(i / j) \cdot(n+1)]}
$$

otherwise $L_{i} / L_{j}=L_{n+1}$.

b) Division as an external operator: $\oslash$. Let $j \neq 0$. We define:

$$
L_{i} \oslash L_{j}=i / j
$$

From the $q$-operators we can directly extend all quantitative fusion rules into their qualitative counterparts by replacing classical operators on numbers by those on linguistic labels defined just above in the formulas. Many useful examples can be found in $[1,3,5,6]$.

\footnotetext{
${ }^{2}$ more $q$-operators with their justifications can be found in [1].

${ }^{3}$ The $q$-multiplication of two linguistic labels defined here can be extended directly to the multiplication of $n>2$ linguistic labels. For example the product of three linguistic label will be defined as $L_{i} \cdot L_{j} \cdot L_{k}=$ $L_{[(i \cdot j \cdot k) /(n+1)(n+1)]}$, etc.

${ }^{4}$ When working with labels, no matter how many operations we have, the best (most accurate) result is obtained if we do only one approximation, and that one should be just at the very end.
}

\section{The 1-Tuple linguistic enriched model}

To take into account the confidence in a linguistic assertions $L_{i}$, we proposed in 2007 [3] a qualitative enriched 1Tuple model, denoted $L_{i}\left(\sigma_{i}^{e}\right)$, where the first component is a standard linguistic label $L_{i}$ and the second component is a confidence factor $\sigma_{i}^{e}$ (either a numerical supporting degree in $[0,1]^{5}$ called Type 1 ; or a qualitative supporting degree taking its value in a given (ordered) set $X$ of linguistic labels, called Type 2). $\sigma_{i}^{e}$ is the confidence one grants to the source when it assigns its qualitative belief $L_{i}$ to a given proposition $A \in G^{\Theta}$. For example, the enriched Type 1 label $L_{1} \triangleq L_{1}(1)$ represents the linguistic variable Good with $100 \%$ confidence, whereas $L_{1}\left(\sigma_{1}^{e}=0.4\right)$ means that the linguistic value $L_{1}$ is discounted by $60 \%$, i.e. we are under confident in $L_{1}$ given by the source. It is important to recall that $\sigma_{i}^{e}$ is related with a confidence measure and does not reflect a positive or negative refinement of the linguistic value itself. That's why $\sigma_{i}^{e}$ and $L_{i}$ are considered as two independent components of the enriched vector $L_{i}\left(\sigma_{i}^{e}\right)$ in the derivations done in [3]. We have shown how to define new qe-operators and how combine qualitative beliefs based on this enriched linguistic 1-Tuple representation model.

\section{The 2-Tuple linguistic model}

In order to keep working with a coarse/reduced set of linguistic labels for maintaining a low computational complexity but for working with a richer information, we adopt here Herrera and Martínez' 2-Tuple model $\left(L_{i}, \sigma_{i}^{h}\right)$ introduced in [2]. $\sigma_{i}^{h}$ is chosen in $\Sigma \triangleq[-0.5 /(n+1), 0.5 /(n+1)), i \in$ $\{1, \cdots, \infty\}$. It is a numerical value of the symbolic translation of our quantitative two-order support $[a, b]$ or of our qualitative two-order support in $X$, say by example $X=$ $\{N B, N M, N S, O, P S, P M, P B\}$. The 2-Tuple model can be justified since each distance between two equidistant labels is $1 /(n+1)$ because of the isomorphism between $L$ and $\{0,1 /(n+1), \ldots, n /(n+1), 1\}$ so that $L_{i}=i /(n+1)$ for all $i=0,1,2, \ldots, n, n+1$. Therefore, we take half to the left and half to the right of each label, so $\sigma_{i}^{h} \in \Sigma$. So a 2-Tuple equidistant linguistic representation model is used to represent the linguistic information by means of 2-Tuple item set $\Pi\left(L, \sigma^{h}\right)$ with $L=\left\{L_{0}, L_{1}, L_{2}, \ldots, L_{n}, L_{n+1}\right\}$ isomorphic to $\{0,1 /(n+1), 2 /(n+1), \ldots, n /(n+1), 1\}$ and the set of qualitative assessments isomorphic to $\boldsymbol{\Sigma}$. This 2-Tuple approach is an intricate/hybrid mechanism of derivation using jointly $L_{i}$ and $\sigma_{i}^{h}$ where $\sigma_{i}^{h}$ is a positive or negative numerical remainder with respect to the labels.

\footnotetext{
${ }^{5}$ In [3], we proposed $\sigma_{i}^{e} \in[0, \infty)$ to allow a possible over confidence factor but since the confidence factor usually comes from statistics it is more natural to take it in $[0,1]$.
} 


\subsection{Symbolic translation}

Let's define the normalized index ${ }^{6} i=\operatorname{round}((n+1) \times$ $\beta)=[(n+1) \times \beta]$, with $i \in[0,(n+1)]$ and $\beta \in[0,1]$, and the Symbolic Translation $\sigma^{h} \triangleq \beta-i /(n+1) \in[-0.5 /(n+$ $1), 0.5 /(n+1))$. Roughly speaking, the symbolic translation of an assessment linguistic value $(n+1) \times \sigma_{i}^{h}$ is a numerical value that supports the difference of information between the (normalized) index obtained from the fusion rule and its closest value in $\{0,1, \ldots, n+1\}$.

\subsection{Useful transformations}

- $\triangle($.$) : conversion of a numerical value into a 2-Tuple$ $\triangle():.[0,1] \rightarrow L \times \boldsymbol{\Sigma}$ is defined by [2]

$$
\triangle(\beta)=\left(L_{i}, \sigma^{h}\right) \triangleq\left\{\begin{array}{l}
L_{i}, \quad i=\operatorname{round}((n+1) \cdot \beta) \\
\sigma^{h}=\beta-i /(n+1), \quad \sigma^{h} \in \mathbf{\Sigma}
\end{array}\right.
$$

Thus $L_{i}$ has the closest index label to $\beta$ and $\sigma^{h}$ is the value of its symbolic translation.

- $\nabla($.$) : conversion of a 2-Tuple into a numerical value$

The inverse/dual function of $\triangle($.$) is denoted \nabla($.$) and$ $\nabla():. L \times \boldsymbol{\Sigma} \rightarrow[0,1]$ is defined by

$$
\nabla\left(\left(L_{i}, \sigma_{i}^{h}\right)\right)=i /(n+1)+\sigma_{i}^{h}=\beta_{i}
$$

It has been proved in [2] that any arithmetic operation commutes with $\triangle($.$) and/or with \nabla($.$) .$

\subsubsection{Useful operators on 2-Tuples}

Let's consider two 2-Tuples $\left(L_{i}, \sigma_{i}^{h}\right)$ and $\left(L_{j}, \sigma_{j}^{h}\right)$, then the following operators ${ }^{7}$ are defined [2].

- Addition of 2-Tuples

$$
\begin{aligned}
&\left(L_{i}, \sigma_{i}^{h}\right)+\left(L_{j}, \sigma_{j}^{h}\right)\left.\equiv \nabla\left(\left(L_{i}, \sigma_{i}^{h}\right)\right)+\left(L_{j}, \sigma_{j}^{h}\right)\right) \\
&=\nabla\left(\left(L_{i}, \sigma_{i}^{h}\right)\right)+\nabla\left(\left(L_{j}, \sigma_{j}^{h}\right)\right)=\beta_{i}+\beta_{j}=\beta_{z} \\
&= \begin{cases}\triangle\left(\beta_{z}\right) & \text { if } \beta_{z} \in[0,1] \\
L_{n+1} & \text { otherwise }\end{cases}
\end{aligned}
$$

- Product of 2-Tuples

\footnotetext{
${ }^{6}$ where round(.) is the rounding operation denoted [.] in our previous $q$-operators [3].

${ }^{7}$ Only those useful for the fusion of 2-Tuple qualitative masses are presented here due to space limitation constraint. More can be found in [2] like negation, comparison, subtraction, etc.
}

$$
\begin{aligned}
& \left(L_{i}, \sigma_{i}^{h}\right) \times\left(L_{j}, \sigma_{j}^{h}\right) \equiv \nabla\left(\left(L_{i}, \sigma_{i}^{h}\right) \times\left(L_{j}, \sigma_{j}^{h}\right)\right) \\
= & \nabla\left(\left(L_{i}, \sigma_{i}^{h}\right)\right) \times \nabla\left(\left(L_{j}, \sigma_{j}^{h}\right)\right)=\beta_{i} \times \beta_{j}=\beta_{p} \equiv \triangle\left(\beta_{p}\right)
\end{aligned}
$$

with $\beta_{p} \in[0,1]$. It can be proved that 2-Tuple addition and product operators are commutative and associative.

- Scalar multiplication of a 2-Tuple

$$
\begin{gathered}
\alpha \cdot\left(L_{i}, \sigma_{i}^{h}\right) \equiv \nabla\left(\alpha \cdot\left(L_{i}, \sigma_{i}^{h}\right)\right)=\alpha \cdot \nabla\left(\left(L_{i}, \sigma_{i}^{h}\right)\right) \\
=\alpha \cdot \beta_{i}=\beta_{\alpha} \equiv\left\{\begin{array}{lc}
\triangle\left(\beta_{\alpha}\right) & \beta_{\alpha} \in[0,1] \\
L_{n+1} & \text { otherwise }
\end{array}\right.
\end{gathered}
$$

- Division of a 2-Tuple by a 2-Tuple

Let's consider two 2-Tuples $\left(L_{i}, \sigma_{i}^{h}\right)$ and $\left(L_{j}, \sigma_{j}^{h}\right)$ with $^{8}$ $\left(L_{i}, \sigma_{i}^{h}\right)<\left(L_{j}, \sigma_{j}^{h}\right)$, then the division is defined as

$$
\begin{aligned}
& \begin{aligned}
\frac{\left(L_{i}, \sigma_{i}^{h}\right)}{\left(L_{j}, \sigma_{j}^{h}\right)} & \equiv \nabla\left(\frac{\left(L_{i}, \sigma_{i}^{h}\right)}{\left(L_{j}, \sigma_{j}^{h}\right)}\right)=\frac{\nabla\left(\left(L_{i}, \sigma_{i}^{h}\right)\right)}{\nabla\left(\left(L_{j}, \sigma_{j}^{h}\right)\right)} \\
& =\frac{\beta_{i}}{\beta_{j}}=\beta_{d} \equiv \triangle\left(\beta_{d}\right) \quad \text { with } \beta_{d} \in[0,1]
\end{aligned} \\
& \text { If }\left(L_{i}, \sigma_{i}^{h}\right) \geq\left(L_{j}, \sigma_{j}^{h}\right), \text { then } \\
& \frac{\left(L_{i}, \sigma_{i}^{h}\right)}{\left(L_{j}, \sigma_{j}^{h}\right)} \equiv \nabla\left(\frac{\left(L_{i}, \sigma_{i}^{h}\right)}{\left(L_{j}, \sigma_{j}^{h}\right)}\right)=\frac{\nabla\left(\left(L_{i}, \sigma_{i}^{h}\right)\right)}{\nabla\left(\left(L_{j}, \sigma_{j}^{h}\right)\right)}=\frac{\beta_{i}}{\beta_{j}} \geq 1
\end{aligned}
$$

and in such case $\frac{\left(L_{i}, \sigma_{i}^{h}\right)}{\left(L_{j}, \sigma_{j}^{h}\right)}$ is set to the maximum label, i.e. $\frac{\left(L_{i}, \sigma_{i}^{h}\right)}{\left(L_{j}, \sigma_{j}^{h}\right)}=\left(L_{n+1}, 0\right) \sim L_{n+1}$.

\section{The 2-Tuple linguistic enriched model}

As for the extension of 1-Tuple model into 1-Tuple enriched model, it is possible to extend the 2-Tuple model into a 2-Tuple enriched model as well, i.e. working with $\left(L_{i}, \sigma_{i}^{h}, \sigma_{i}^{e}\right)$. This can be done pretty easily but this will be not reported in this paper due to the space limitation constraint. This will be subject to a forthcoming publication.

\section{Fusion of qualitative beliefs with 2-Tuple}

From the 2-Tuple model of qualitative beliefs and the previous operators, we are able to extend the PCR5 and Demspter-Shafer's (DS) fusion rules in the qualitative domain in a more precise way than done before. The qualitative belief mass/assignment (qba) $q_{2} m(\cdot)$ based on 2-Tuple

\footnotetext{
${ }^{8}$ The comparison operator is defined in [2].
} 
representation is defined as $q_{2} m(\cdot): G^{\Theta} \rightarrow L \times \sigma^{\mathbf{h}}$ such that $q_{2} m(\emptyset)=\left(L_{0}, 0\right)$ and $\sum_{A \in G^{\Theta}} q_{2} m(A)=\left(L_{n+1}, 0\right)$. The $q_{2}$-extensions of PCR5 (1) and Demspter-Shafer's fusion rules [4] for two sources on a frame $\Theta$ based on the 2-Tuple operators are then given by ${ }^{9}$ :

- $q_{2}$-extension of PCR5 fusion rule: $q_{2} m_{P C R 5}(\emptyset)=$ $\left(L_{0}, 0\right)$ and $\forall X \in G^{\Theta} \backslash\{\emptyset\}$

$$
\begin{array}{r}
q_{2} m_{P C R 5}(X)=q_{2} m_{12}(X)+ \\
\sum_{\substack{Y \in G^{\Theta} \backslash\{X\} \\
X \cap Y=\emptyset}}\left[\frac{q_{2} m_{1}(X)^{2} q_{2} m_{2}(Y)}{q_{2} m_{1}(X)+q_{2} m_{2}(Y)}+\right. \\
\left.\frac{q_{2} m_{2}(X)^{2} q_{2} m_{1}(Y)}{q_{2} m_{2}(X)+q_{2} m_{1}(Y)}\right]
\end{array}
$$

where $q_{2} m_{12}(X)$ corresponds to the qualitative $q_{2}$ extension of the conjunctive consensus.

- $q_{2}$-extension of Dempster-Shafer fusion rule: $q_{2} m_{D S}(\emptyset)=\left(L_{0}, 0\right)$ and $\forall X \in 2^{\Theta} \backslash\{\emptyset\}$

$$
q_{2} m_{D S}(X)=\frac{\sum_{\substack{X_{1}, X_{2} \in 2 \Theta \\ X_{1} \cap X_{2}=X}} q_{2} m_{1}\left(X_{1}\right) q_{2} m_{2}\left(X_{2}\right)}{L_{n+1}-K_{12}}
$$

where the total degree of qualitative conflict is given by $K_{12} \triangleq \sum_{\substack{X_{1}, X_{2} \in 2^{\Theta} \\ X_{1} \cap X_{2}=\emptyset}} q_{2} m_{1}\left(X_{1}\right) q_{2} m_{2}\left(X_{2}\right)$.

It is important to note that the addition, the product and the division operators involved in the previous formulas are the 2-Tuple operators defined in previous section. The extensions (14) and (15) are well justified since every 2-Tuple $\left(L_{i}, \sigma_{i}^{h}\right)$ can be mapped into a unique numerical value $\beta$ corresponding to it which makes $q_{2 P C R 5}$ and $q_{2 D S}$ equivalent to PCR5 and DS because of $\Delta($.$) function.$

\section{Example of fusion of qualitative beliefs}

Let's consider an investment corporation which must choose one of three projects in $\Theta=\left\{\theta_{1}, \theta_{2}, \theta_{3}\right\}$ (assume here that Shafer's model holds for simplicity) to invest through two consulting departments. A set of qualitative values are used to describe the opinions of two consulting companies, i.e. I $\mapsto$ Impossible, EU $\mapsto$ Extremely-Unlikely, VLC $\mapsto$ Very-Low-Chance, LLC $\mapsto$ Little-Low-Chance, $\mathrm{SC} \mapsto$ Small-Chance, IM $\mapsto$ IT-May, MC $\mapsto$ MeanfulChance, LBC $\mapsto$ Little-Big-Chance, BC $\mapsto$ Big-Chance, $\mathrm{ML} \mapsto$ Most-likely, $\mathrm{C} \mapsto$ Certain. So, we consider the set of ordered linguistic labels $L=\left\{L_{0} \equiv I, L_{1} \equiv E U, L_{2} \equiv\right.$

\footnotetext{
${ }^{9}$ These formulas can obviously be extended directly for $N>2$ sources.
}

$V L C, L_{3} \equiv L L C, L_{4} \equiv S C, L_{5} \equiv I M, L_{6} \equiv M C, L_{7} \equiv$ $\left.L B C, L_{8} \equiv B C, L_{9} \equiv M L, L_{10} \equiv C\right\}$ and in this example $n=9$.

The opinions of the two consulting companies/sources are given in Table 1

\begin{tabular}{|c|ccc|}
\hline$m(\cdot)$ & $\theta_{1}$ & $\theta_{2}$ & $\theta_{3}$ \\
\hline Source no 1 & $\left(L_{4}, 0.3\right)$ & $\left(L_{3},-0.3\right)$ & $\left(L_{3}, 0\right)$ \\
Source no 2 & $\left(L_{5}, 0\right)$ & $\left(L_{2}, 0.1\right)$ & $\left(L_{3},-0.1\right)$ \\
\hline
\end{tabular}

\section{Table 1. Qualitative 2-Tuple belief masses}

Following PCR5, the masses of the partial conflicts $\theta_{1} \cap$ $\theta_{2}, \theta_{1} \cap \theta_{3}$ and $\theta_{2} \cap \theta_{3}$ are redistributed to those belief masses involved in these conflicts according to (14). One gets:

$$
\begin{aligned}
& q_{2} m_{x A 1}\left(\theta_{1}\right)=\frac{\left(L_{4}, 0.3\right) \times\left(L_{1},-0.097\right)}{\left(L_{6}, 0.4\right)} \approx\left(L_{1},-0.393\right) \\
& q_{2} m_{y A 1}\left(\theta_{2}\right)=\frac{\left(L_{2}, 0.1\right) \times\left(L_{1},-0.097\right)}{\left(L_{6}, 0.4\right)} \approx\left(L_{0}, 0.296\right) \\
& q_{2} m_{x B 1}\left(\theta_{1}\right)=\frac{\left(L_{5}, 0\right) \times\left(L_{1}, 0.35\right)}{\left(L_{8},-0.3\right)} \approx\left(L_{1},-0.123\right) \\
& q_{2} m_{y B 1}\left(\theta_{2}\right)=\frac{\left(L_{3},-0.3\right) \times\left(L_{1}, 0.35\right)}{\left(L_{8},-0.3\right)} \approx\left(L_{0}, 0.473\right) \\
& q_{2} m_{x A 2}\left(\theta_{1}\right)=\frac{\left(L_{4}, 0.3\right) \times\left(L_{1}, 0.247\right)}{\left(L_{7}, 0.2\right)} \approx\left(L_{1},-0.255\right) \\
& q_{2} m_{y A 2}\left(\theta_{3}\right)=\frac{\left(L_{3},-0.1\right) \times\left(L_{1}, 0.247\right)}{\left(L_{7}, 0.2\right)} \approx\left(L_{1},-0.497\right)
\end{aligned}
$$

and similarly, one has $q_{2} m_{x B 2}\left(\theta_{1}\right) \approx\left(L_{1},-0.062\right)$, $q_{2} m_{y B 2}\left(\theta_{3}\right) \approx\left(L_{1},-0.437\right), q_{2} m_{x A 3}\left(\theta_{2}\right) \approx\left(L_{0}, 0.377\right)$, $q_{2} m_{y A 3}\left(\theta_{3}\right) \approx\left(L_{0}, 0.405\right), q_{2} m_{x B 3}\left(\theta_{2}\right) \approx\left(L_{0}, 0.259\right)$ and $q_{2} m_{y B 3}\left(\theta_{3}\right) \approx\left(L_{0}, 0.370\right)$. Thus, one finally gets:

$$
\begin{gathered}
q_{2} m_{P C R 5}\left(\theta_{1}\right)=q_{2} m_{12}\left(\theta_{1}\right)+q_{2} m_{x A 1}\left(\theta_{1}\right)+ \\
q_{2} m_{x B 1}\left(\theta_{1}\right)+q_{2} m_{x A 2}\left(\theta_{1}\right)+q_{2} m_{x B 2}\left(\theta_{1}\right) \approx\left(L_{5}, 0.315\right) \\
q_{2} m_{P C R 5}\left(\theta_{2}\right)=q_{2} m_{12}\left(\theta_{2}\right)+q_{2} m_{y A 1}\left(\theta_{2}\right)+ \\
q_{2} m_{y B 1}\left(\theta_{2}\right)+q_{2} m_{x A 3}\left(\theta_{2}\right)+q_{2} m_{x B 3}\left(\theta_{2}\right) \approx\left(L_{2},-0.026\right) \\
q_{2} m_{P C R 5}\left(\theta_{3}\right)=q_{2} m_{D S m C}\left(\theta_{3}\right)+q_{2} m_{y A 2}\left(\theta_{3}\right)+ \\
q_{2} m_{y B 2}\left(\theta_{3}\right)+q_{2} m_{y A 3}\left(\theta_{3}\right)+q_{2} m_{y B 3}\left(\theta_{3}\right) \approx\left(L_{3},-0.289\right)
\end{gathered}
$$

Since $q_{2} m_{P C R 5}\left(\theta_{1}\right)>q_{2} m_{P C R 5}\left(\theta_{2}\right)$ and $q_{2} m_{P C R 5}\left(\theta_{1}\right)$ $>q_{2} m_{P C R 5}\left(\theta_{3}\right)$, the investment corporation must invest in the project $\theta_{1}$. Using DS fusion (15), the total conflict is $q K_{12}=q_{2} m_{12}\left(\theta_{1} \cap \theta_{2}\right)+q_{2} m_{12}\left(\theta_{1} \cap \theta_{3}\right)+q_{2} m_{12}\left(\theta_{3} \cap\right.$ $\left.\theta_{2}\right)=\left(L_{6}, 0.413\right)$. Thus $q_{2} m_{D S}(\emptyset) \triangleq\left(L_{0}, 0\right)$ and

$$
\begin{aligned}
q_{2} m_{D S}\left(\theta_{1}\right) & =\frac{q_{2} m_{12}\left(\theta_{1}\right)}{L_{10}-q K_{12}}=\frac{\left(L_{2}, 0.15\right)}{L_{10}-\left(L_{6}, 0.413\right)} \\
& \approx\left(L_{6},-0.006\right)
\end{aligned}
$$




$$
\begin{aligned}
q_{2} m_{D S}\left(\theta_{2}\right) & =\frac{q_{2} m_{12}\left(\theta_{2}\right)}{L_{10}-q K_{12}}=\frac{\left(L_{1},-0.413\right)}{L_{10}-\left(L_{6}, 0.413\right)} \\
& \approx\left(L_{2},-0.419\right) \\
q_{2} m_{D S}\left(\theta_{3}\right) & =\frac{q_{2} m_{12}\left(\theta_{3}\right)}{L_{10}-q K_{t 12}}=\frac{\left(L_{1},-0.13\right)}{L_{10}-\left(L_{6}, 0.413\right)} \\
& \approx\left(L_{2}, 0.425425\right)
\end{aligned}
$$

$q_{2} m_{D S}\left(\theta_{1}\right)$ is still larger than $q_{2} m_{D S}\left(\theta_{2}\right)$ and $q_{2} m_{D S}\left(\theta_{3}\right)$ and the first project is also chosen to invest based on DS rule. The final decision is same as the previous one based on $q_{2} m_{P C R 5}$. However, when the total conflict increases up to $L_{10}$, then $q_{2} m_{D S}$ results for decision-making can become counter-intuitive and yield to wrong decision (see [5] for counter examples of DS rule).

From our analysis, the following advantages can be drawn on the usefulness of 2-Tuple representation coupled with DSmT framework and PCR5.

a) High Precision: Based on 2-Tuples, $q_{2}$ operators provide a higher precision than with 1 -Tuples $q_{1}$ and $q_{e}$ operators because for every 2-Tuple $\left(L_{i}, \alpha_{i}\right)$, there is a unique $\beta \in[-(n+1), n+1]$ corresponding to it and thus one doesn't lose information in the computations.

b) Wide adaptive capacity: Since 2 -Tuples $\left\{\left(L_{0}, \alpha_{0}\right), \ldots\right.$, $\left.\left(L_{n+1}, \alpha_{n+1}\right)\right\}$ express a continuous qualitative belief, it is equivalent to real number. So all quantitative fusion rules and belief conditioning rules can be used directly in this framework. As already proved for fusion of qualitative belief masses, it is expected that $q_{2} m_{P C R 5}$ approach will outperform $q_{2} m_{D S}$, specially in all high conflicting situations.

c) Low complexity: Since the addition and multiplication operators on 2-Tuple are commutative and associative, while $q_{1}$ and $q_{e}$ models depend on the order/approximation of the operations carried out ${ }^{10}$ the fusion based on 2-Tuple theoretically works better than with $q_{1}$ and $q_{e}$ models.

\section{Conclusion}

In this paper, we have proposed a new approach for combining uncertain qualitative beliefs based on 2 -Tuple linguistic labels and DSmT-PCR5 fusion rule. The main idea was to refine the granularity of linguistic information without extending the original set of linguistic labels to keep

\footnotetext{
${ }^{10}$ This dependency can be however diminished if the approximation is done at the very end step.
}

a low computational complexity. For achieving such purpose, Herrera-Martinez 2-Tuple representation model has been adopted and a new set of qualitative operators for 2Tuples has been defined ( $q_{2}$-operators). We have shown that this method is a generalization and an improvement of other method based either on $q_{1}$ operators (for 1-Tuple/pure linguistic labels) or on qe operators. $q_{2}$-operators are hybrid operators which work jointly on the first component (index of the label) and on the second component (positive or negative refinement of the label) of the 2-Tuples. On a very simple example, we have shown how we can combine qualitative beliefs with these 2-Tuples and $q_{2}$-operators. The results obtained are more precise than those based on $q_{1}$ or $q e$-operators since no approximation is done during derivations and all the information is used in the fusion process. This approach is an interesting bridge between qualitative and quantitative reasoning under uncertainty. Applications of this approach in robotics are under progress and will be presented in a forthcoming publication.

\section{Acknowledgment}

This work is partially supported by The National High Technology Research and Development Program of China (863 Program, NO:2006AA040202).

\section{References}

[1] J. Dezert, F. Smarandache, "A new probabilistic transformation of belief mass assignment", In Proc. of Fusion 2008, Cologne, Germany, July 2008.

[2] F. Herrera , L. Martínez, "A 2-Tuple fuzzy linguistic representation model for computing with words." IEEE Trans. on Fuzzy Systems, 8 (6), pp. 746-752, 2000.

[3] X. Li, X. Huang, J. Dezert and F. Smarandache, "Enrichment of Qualitative Beliefs for Reasoning under Uncertainty.” Fusion 2007 Int. Conf., July 2007.

[4] G. Shafer, "A Mathematical Theory of Evidence", Princeton University Press, Princeton, NJ, 1976.

[5] F. Smarandache and J. Dezert (Editors), "Advances and Applications of DSmT for Information Fusion”, Vol.1 \& 2, American Research Press, Rehoboth, 2004 \& 2006. http://fs.gallup.unm.edu//DSmT.htm.

[6] F. Smarandache, J. Dezert, "Information Fusion Based on New Proportional Conflict Redistribution Rules", Proc. of Fusion 2005, July 2005.

[7] F. Smarandache, J. Dezert, "Qualitative Belief Conditioning Rules”, Fusion 2007 Int. Conf., July 2007. 